\title{
Enhancing the Efficiency of Spectrum Occupancy Measurement and Evaluation with Signal Lifetime
}

\author{
Xueling Zhang a, Liang Chen, JiaJia Chen and Fuyin Zhao \\ The State Radio Monitoring Center, Beijing 100000, China. \\ azhangxueling@srrc.org.cn
}

\begin{abstract}
The usage of the spectrum and the trends in its demand is both critical to a good spectrum management and necessary to frequency allotments and assignments. In most cases automatic occupancy measurement based on traditional method is well suited but there still exists some deficiency. With a long-term monitoring experience, we put forward the concept of signal lifetime and propose a new method of measuring spectrum occupancy based on signal lifetime system. Experimental results show that the advantages of signal lifetime system in occupancy measuring greatly enhance the efficiency.
\end{abstract}

Keywords: Signal Lifetime, spectrum occupancy, measurement.

\section{Introduction}

The increasing demand of radio communication services requires the most efficient usage of the radio frequency spectrum, especially in $5 \mathrm{G}$ communication. We all know that the results of spectrum occupancy measurements are used to provide important inputs into establishment of the degree of efficiency of spectrum usage all over the world. [1] But the existing measurements are time consuming. In order to support increasing throughput requirements of $5 \mathrm{G}$, large quantities of new spectrum (5G NR frequency bands) have been allocated to 5G, particularly in mm Wave bands. [2] With more spectrum usage, a more efficient measurement and evaluation of spectrum occupancy will be required. In group study which has been proceeded for over a year, we put forward the concept of signal lifetime and set up a signal lifetime system. In the follow-up study using improved big data algorithm, signal lifetime system can also be used in spectrum occupancy prediction.

In the following paper, first we define signal lifetime and explain how to measure spectrum occupancy using the signal lifetime system. Then we make an experiment of evaluating the occupancy of a certain spectrum by traditional method and by our new method. Finally, by comparing the two methods, conclusions are given.

\section{Signal Lifetime}

\subsection{What is Signal Lifetime}

In the field of radio monitoring, it's important to know when a signal occurred and when it will occur. The occurrence tells the regular feature of emission spectra. It's very likely that there would be more than one signal in a spectrum, so it's difficult to measure the occupancy considering every single signal. In this case, signal lifetime is defined as the existed living time and the predicted living time of a particular signal. Every signal has a signal lifetime. We study the signals by analyzing monitoring data and then storing them in signal lifetime system.

In our system, there are five procedures: data acquisition, signal search and finding, signal living status record, signal emission analysis and signal predicting.

Based on the background noise we calculate the threshold value dynamically to search signals. In this process we use two new algorithms named one-frame moving average and multi-frame moving average, which are better than the current methods.

\subsection{Signal Lifetime Storage}

The system calculates signal lifetime and the signal occupancy every 15 minutes and stores the results in the database. After getting the spectrum frame data in real time from a receiver or spectrum 
analyzer, signal lifetime system identifies signals from the data and calls the interface provided by database to find new signals.

Based on these results, the system merge signals and calculate signal lifetime in real-time.

If a signal exists in both the previous frame and the current frame,

Signal lifetime (milliseconds) $+=$ (current time - last frame time)

Upon arrival time of a period of 15 minutes, the signal occupancy is calculated as follows:

Signal occupancy $=$ signal lifetime $/(15 * 60 * 1000)$

At the same time, the system calculates frequency occupancy in real time according to the signal bandwidth.

If a certain frequency falls into at least one signal bandwidth in both current frame and the previous frame,

Frequency occupied time (milliseconds) $+=$ (current time - last frame time)

Upon arrival time of a period of 15 minutes, the frequency occupancy is calculated as follows:

Frequency occupancy $=$ frequency occupied time/ $(15 * 60 * 1000)$

For the short-wave frequency band, the frequency occupation is calculated with a minimum resolution unit of $1 \mathrm{KHz}$, while for the ultra-short wave band, the frequency occupation is calculated with a minimum resolution unit of $10 \mathrm{KHz}$.

\subsection{Signal Lifetime Statistics}

The signal lifetime is calculated and stored for every single signal. The system list a signal ID selected through a certain frequency range, and then calculate the signals one by one.

The statistic of signal lifetime is based on the signal occupancy table, which is put into storage every 15 minutes. In order to calculate the signal lifetime, first query all the original data according to the query date, and then sort the statistical results according to the time interval (such as 15 minutes, 1 hour, 1 day, etc.) If the time interval is 15 minutes, then we observe whether or not the signal ID is occupied within the 15 minutes. If the time interval is 1 hour, then we observe whether or not the signal ID is occupied within this whole hour. For example, when the time interval is from 13:00 to 14:00, as long as there is occupancy in any of the four minimum segments (13:00-13:15, 13:15-13:30, 13:30-13:45 and 13:45-14:00), the signal is regarded to be occupied within this hour.

Once the signal identification list is generated, the algorithm is called to calculate and store the signal occupancy, of which the process is shown in figure 1.

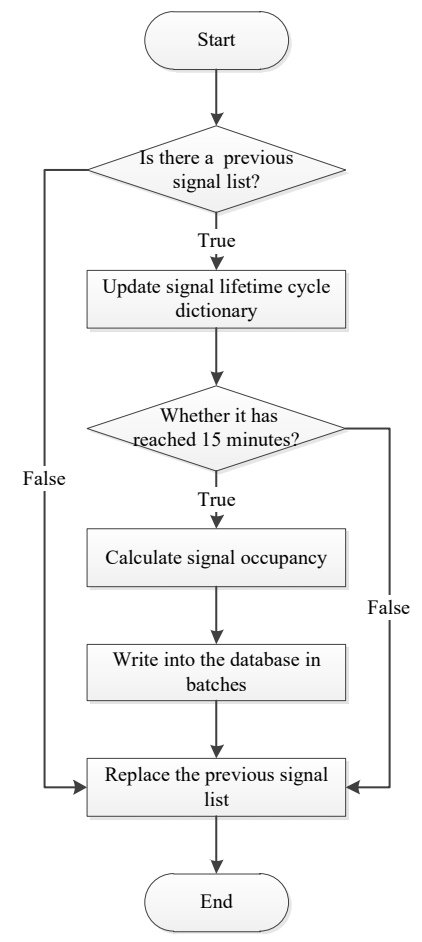

Fig 1. Flow chart of signal occupancy calculation 
Description of the flow chart:

Update signal lifetime cycle dictionary: lifecycle dictionary contains all the signal lifetime data within a computation period (15 minutes). The Key is the ID of a signal; the Value is the survival time. After traversing previous signal list, add the survival time with the time interval between two sampling, and then update the result to the signal lifetime cycle dictionary.

Calculate signal occupancy: signals occupancy $=$ signal lifetime/total time $(15$ minutes $) * 100 \%$

Write into the database in batches: after the calculation, update all the signal occupancy in a same transaction, and at the same time update them to the "occupiedratetrack" table, which is used to represent whether the calculation cycle data is valid.

Replace the previous signal list: replace the previous signal list with the new signal list.

Based on these statistical results, several different ways of showing are designed. For instance, in intersection display the lifetimes are merged in adjacent time blocks, and in combination display when the lifetime has same regulation within a certain time interval in different days or even months it will be showed as survival.

The presentation system should, as a minimum, contain the location of the monitoring site, date and period of measurement, frequency, type of user(s), threshold level used, occupancy in the busy hour and revisit period. [3]

\subsection{Spectrum Occupancy Measurement with Signal Lifetime}

As in 2.3 we've known how to measure the signal occupancy according to signal lifetime. Spectrum occupancy can be calculated depend on the signal occupancy. The situations are as below:

If the target frequency is exactly the same of the of a signal center frequency, then the frequency occupancy is equal to the signal occupancy.

If there is a frequency deviation between the target frequency and a signal center frequency and it is within the bandwidth of the signal, then the frequency occupancy is equal to the signal occupancy.

If there is a frequency deviation between the target frequency and a signal center frequency and it is within the bandwidth of a several adjacent signals, then the frequency occupancy is the combination of the several adjacent signals with the ratio of total time interval.

If there is a frequency deviation between the target frequency and a signal center frequency and it isn't in any signal bandwidth, then the frequency occupancy is 0 .

According to the channel covered by the signal's bandwidth, divide its number by the total channel number to get the spectrum occupancy. This method has an advantage that it needs less storage and less calculation.

Spectrum occupancy measurement algorithm is the same as signal occupancy measurement in 2.3, in which the Key is the frequency, and of which the survival time has only one increment when is occupied by several signals.

An experiment of spectrum occupancy measurement

Traditionally, frequency channel occupancy measurements are performed with a receiver or spectrum analyser. After storing the signal strength of each frequency step, a certain method is needed to determine the percentage of time in which the signal is above a certain threshold level, which should be preset. If a signal is received above the threshold level, the channel is considered to be occupied. The results can be stored every 5, 15, 30 or $60 \mathrm{~min}$ as required. From this data it is possible to generate presentations based on tables, textual graphs, line/bar graphs and maps. [4]

Using traditional measurement method

Equipment: a high-performance receiver;

Monitoring time: 2018-8-17 12:04 to 2018-8-20 11:22;

Monitoring method: 24 hours*3days;

Scan step: $0.5 \mathrm{kHz}$;

Threshold level: $15 \mathrm{~dB} \mu v / \mathrm{m}, 20 \mathrm{~dB} \mu v / \mathrm{m}, 25 \mathrm{~dB} \mu v / \mathrm{m}, 30 \mathrm{~dB} \mu v / \mathrm{m}$.

After 3 days monitoring, the receiver stored 1,584,264 records. In order to measure the spectrum, 6 testing frequency are chosen: $6.035 \mathrm{MHz}, 6.075 \mathrm{MHz}, 7.276 \mathrm{MHz}, 7.277 \mathrm{MHz}, 8.25 \mathrm{MHz}$, and $8.055 \mathrm{MHz}$. The result is shown in table 1. 
Table 1. Results of 6 testing frequency with the traditional method

\begin{tabular}{ccccc}
\hline Frequency & $\begin{array}{c}\text { Occupancy } \\
\text { (Threshold level: } 15 \\
\mathrm{~dB} \mu \nu / \mathrm{m})\end{array}$ & $\begin{array}{c}\text { Occupancy } \\
\text { (Threshold level: } 20 \\
\mathrm{~dB} \mu \nu / \mathrm{m})\end{array}$ & $\begin{array}{c}\text { Occupancy } \\
\text { (Threshold level: } 25 \\
\mathrm{~dB} \mu v / \mathrm{m})\end{array}$ & $\begin{array}{c}\text { Occupancy } \\
\text { (Threshold level: } 30 \\
\mathrm{~dB} \mu \nu / \mathrm{m})\end{array}$ \\
\hline $\begin{array}{c}6.035 \mathrm{MHz} \\
6.075\end{array}$ & $87.12 \%$ & $83.71 \%$ & $79.17 \%$ & $68.94 \%$ \\
$\mathrm{MHz}$ & $89.39 \%$ & $85.98 \%$ & $83.71 \%$ & $74.62 \%$ \\
7.276 & $88.26 \%$ & $81.44 \%$ & $72.73 \%$ & $62.12 \%$ \\
$\mathrm{MHz}$ & & & & $16.67 \%$ \\
7.277 & $48.48 \%$ & $40.15 \%$ & $29.55 \%$ & $0 \%$ \\
$\mathrm{MHz}$ & $3.03 \%$ & $0.38 \%$ & $0 \%$ & $0 \%$ \\
$8.25 \mathrm{MHz}$ & $3.41 \%$ & $0 \%$ & $0 \%$ & 0 \\
$8.36 \mathrm{MHz}$ & & & & \\
\hline
\end{tabular}

\subsection{Using Signal Lifetime System}

The same bandwidth $(6 \mathrm{MHz}-9 \mathrm{MHz})$ is studied with signal lifetime system, and a signal list is given in the bandwidth. There are only five signals in this bandwidth, and some of their most important parameters are showed in table 2.

Table 2. signal list in the $6 \mathrm{MHz}-9 \mathrm{MHz}$ by signal lifetime system

\begin{tabular}{|c|c|c|c|c|c|}
\hline $\begin{array}{c}\text { Center } \\
\text { frequency }\end{array}$ & bandwidth & Signal survival time & $\begin{array}{c}\text { Signal } \\
\text { Lifetime }\end{array}$ & $\begin{array}{l}\text { Total living } \\
\text { time }\end{array}$ & $\begin{array}{c}\text { Signal } \\
\text { occupancy }\end{array}$ \\
\hline $6.035 \mathrm{MHz}$ & $12 \mathrm{kHz}$ & $\begin{array}{l}07: 00-07: 15 ; 07: 15-07: 30 ; \ldots .07: 45- \\
08: 00 ; \ldots 13: 15-13: 30 ; \ldots 23: 45-24: 00\end{array}$ & $\begin{array}{l}07: 00- \\
24: 00\end{array}$ & 18hours & $75 \%$ \\
\hline $6.075 \mathrm{MHz}$ & $6 \mathrm{kHz}$ & $\begin{array}{l}06: 00-06: 15 ; 06: 15-06: 30 ; \ldots .07: 45- \\
08: 00 ; \ldots 13: 15-13: 30 ; \ldots 23: 45-24: 00\end{array}$ & $\begin{array}{l}06: 00- \\
24: 00\end{array}$ & 19hours & $79.16 \%$ \\
\hline 7.22MHz & $9 \mathrm{kHz}$ & $\begin{array}{l}05: 00-05: 15 ; 05: 15-05: 30 ; \ldots .07: 45- \\
08: 00 ; \ldots 13: 15-13: 30 ; \ldots 23: 45-24: 00\end{array}$ & $\begin{array}{l}05: 00- \\
24: 00\end{array}$ & 20hours & $83.33 \%$ \\
\hline $7.275 \mathrm{MHz}$ & $12 \mathrm{kHz}$ & $\begin{array}{l}04: 30-04: 45 ; 04: 45-05: 00 ; \ldots .07: 45- \\
08: 00 ; \ldots 13: 15-13: 30 ; \ldots 23: 45-24: 00\end{array}$ & $\begin{array}{l}04: 30- \\
24: 00\end{array}$ & 20.5hours & $85.42 \%$ \\
\hline $7.23 \mathrm{MHz}$ & $9 \mathrm{kHz}$ & $\begin{array}{l}05: 00-05: 15 ; 05: 15-05: 30 ; \ldots . .07: 45- \\
08: 00 ; \ldots 13: 15-13: 30 ; \ldots 23: 45-24: 00\end{array}$ & $\begin{array}{l}05: 00- \\
24: 00\end{array}$ & 20hours & $83.33 \%$ \\
\hline
\end{tabular}

In order to compare results, the same testing frequency as in 3.1 was chosen. The results are shown in table 3.

Table 3. Results of 6 testing frequency with signal lifetime system

\begin{tabular}{ccccc}
\hline Frequency & $\begin{array}{c}\text { Occupancy } \\
\text { (Threshold level: } 15 \\
\mathrm{~dB} \mu v / \mathrm{m})\end{array}$ & $\begin{array}{c}\text { Occupancy } \\
\text { (Threshold level: } 20 \\
\mathrm{~dB} \mu v / \mathrm{m})\end{array}$ & $\begin{array}{c}\text { Occupancy } \\
\text { (Threshold level: } 25 \\
\mathrm{~dB} \mu v / \mathrm{m})\end{array}$ & $\begin{array}{c}\text { Occupancy } \\
\text { (Threshold level: } 30 \\
\mathrm{~dB} \mu v / \mathrm{m})\end{array}$ \\
\hline $\begin{array}{c}7.035 \mathrm{MHz} \\
6.075\end{array}$ & $75 \%$ & $75 \%$ & $75 \%$ & $75 \%$ \\
$\mathrm{MHz}$ & $79.16 \%$ & $79.16 \%$ & $79.16 \%$ & $79.16 \%$ \\
7.276 & & & & \\
$\mathrm{MHz}$ & $85.42 \%$ & $85.42 \%$ & $85.42 \%$ & $85.42 \%$ \\
7.277 & & & & \\
$\mathrm{MHz}$ & $42.71 \%$ & $42.71 \%$ & $21.36 \%$ & $0 \%$ \\
$8.25 \mathrm{MHz}$ & $0 \%$ & $0 \%$ & $0 \%$ & $0 \%$ \\
$8.36 \mathrm{MHz}$ & $0 \%$ & $0 \%$ & $0 \%$ & $0 \%$ \\
\hline
\end{tabular}

\subsection{Analysis and Comparison of the two Methods}

According to the experiment above, it isn't difficult to find that the traditional method to measure spectrum occupancy has a high accuracy, but it also has many disadvantages, while the new method preforms better than traditional method in many aspects, such as the requirement to receiver is lower, data storage is smaller, overhead is smaller, efficiency is higher and prediction is possible. The comparison table is shown in table 4. 
Table 4. A comparison table of the two methods

\begin{tabular}{|c|c|c|}
\hline $\begin{array}{c}\text { Key } \\
\text { Performance }\end{array}$ & Traditional measurement method & A new method with signal lifetime system \\
\hline Equipment & $\begin{array}{l}\text { Require high performance } \\
\text { receiver }\end{array}$ & No special requirements \\
\hline Data storage & $\begin{array}{c}\text { Large storage } \\
\text { For example, to store a } \\
\text { bandwidth of } 3 \mathrm{MHz} \text { spectrum, } \\
\text { needs } \\
3 * 24 \text { hours: more than } 1.5 \mathrm{M} \text { data } \\
1 \text { month: more than } 15 \mathrm{Mdata} \\
1 \text { year: more than } 183 \mathrm{M} \text { data }\end{array}$ & $\begin{array}{c}\text { Small storage } \\
\text { For example, to store a bandwidth of } 3 \mathrm{MHz} \\
\text { spectrum, needs } \\
3 * 24 \text { hours: less than } 1 \mathrm{k} \text { data } \\
1 \text { month: less than } 1 \mathrm{k} \text { data } \\
1 \text { year: less than } 1 \mathrm{k} \text { data }\end{array}$ \\
\hline Overhead & A big run-time overhead & A small run-time overhead \\
\hline Accuracy & High accuracy & Moderate accuracy \\
\hline Efficiency & Low efficiency & High efficiency \\
\hline Prediction & $\begin{array}{l}\text { Can't predict future spectrum } \\
\text { occupancy. }\end{array}$ & $\begin{array}{l}\text { Based on signal lifetime, using the big data } \\
\text { algorithm, a prediction of spectrum occupancy can } \\
\text { be made. }\end{array}$ \\
\hline
\end{tabular}

\section{Summary}

In summary, we put forward the concept of signal lifetime and propose a new method based on signal lifetime system. Traditionally, the spectrum occupancy is calculated by scanning a certain bandwidth with a preset frequency step, which is costly and often needs a large storage. Through an experiment we conduct our new method and prove that it significantly enhances the efficiency of spectrum occupancy by downsizing the data storage and reducing the calculation time, also it can be used for prediction of spectrum occupancy. It's expected that signal lifetime will play an indispensable role in the improvement of spectrum occupancy evaluation in $5 \mathrm{G}$ era.

\section{Acknowledgments}

This work is supported by the National Science and Technology Major Project under Grants No. $2015 Z X 03002008$.

\section{References}

[1]. Hong shun Zhou. Handbook of spectrum monitoring. People's posts and telecommunications press. 2006, p.1-3.

[2]. 5G Americas White Paper on 5G Spectrum Recommendations- April 2017.

[3]. SM Series Spectrum management. Spectrum occupancy measurements and evaluation. Recommendation ITU-R SM.1880-2(09/2017).

[4]. Information on: www.itu.int. 